\title{
Optimal Cotangential Orbital Transfer Maneuvers
}

\author{
M.A. Sharaf \\ Department of Astronomy, Faculty of Science, \\ King Abdulaziz University, Jeddah, Saudi Arabia
}

\begin{abstract}
In this paper, optimal two impulses cotangential orbital transfer maneuvers is developed for which: 1. Both the inner and transfer orbits are of arbitrary shapes (circular, elliptic, parabolic or hyperbolic). 2. The two impulses are applied tangentially at two general points $r_{1}$ and $r_{2}$ not necessarily to be applied at the apsides.

The solution of the problem is thus; the determination of, the semimajor axis $\mathrm{a}_{\mathrm{T}}$ and the eccentricity $\mathrm{e}_{\mathrm{T}}$ of the transfer orbit, and the total impulse for the complete two impulse transfer at $r_{1}$ and $r_{2}$ which gives minimum velocity increment. Some numerical applications are also included.
\end{abstract}

Keywords: Astrodynamics - orbital maneuvers - trajectory optimization.

\section{Introduction}

Despite of the theories and a lot of numerical work developed in the field of orbital maneuvers e.g. [Chobotov 1996, Villac and Scheeres 2003, Lin $2007^{[1-3]}$, most of the transfers assumed an underlying two body model and investigate the different ways of transferring from one orbit of a given fixed type (elliptic, parabolic, or hyperbolic) to another orbit, also of a given but not necessary of the same type as the first orbit.

During space mission all types of the two body motion (elliptic, parabolic, or hyperbolic) appear. For examples the escape from the departure planet and the capture by the target planet involve hyperbolic orbits, while the intermediate stage of the mission commonly depicted as a heliocentric ellipse, may also be heliocentric parabola or hyperbola. In addition, in some systems, the type of an orbit is occasionally changed by 
perturbing forces during finite interval of time. Thus far we have been obliged to use different functional representations for motion depending upon the energy state (elliptic, parabolic, or hyperbolic) and a simulation code must then contain branching to handle a switch from one state to another. In cases where this switching is not smooth, branching can occur many times during a single integration time-step causing some numerical "chatter". Consequently, universal formulations are desperately needed so that, orbit transfers will be free of the troubles, since a single functional representation suffices to describe all possible states.

In the present paper, universal cotangential transfer maneuvers based on the classical constraint minimization approach [e.g. Escobal 1968] $]^{[4]}$, will be developed for which:

1. Both the inner and transfer orbits are of arbitrary shapes (circular, elliptic, parabolic or hyperbolic).

2. The two impulses are applied tangentially at two general points $r_{1}$ and $r_{2}$ (see Fig. 1), not necessarily to be applied at the apsides.

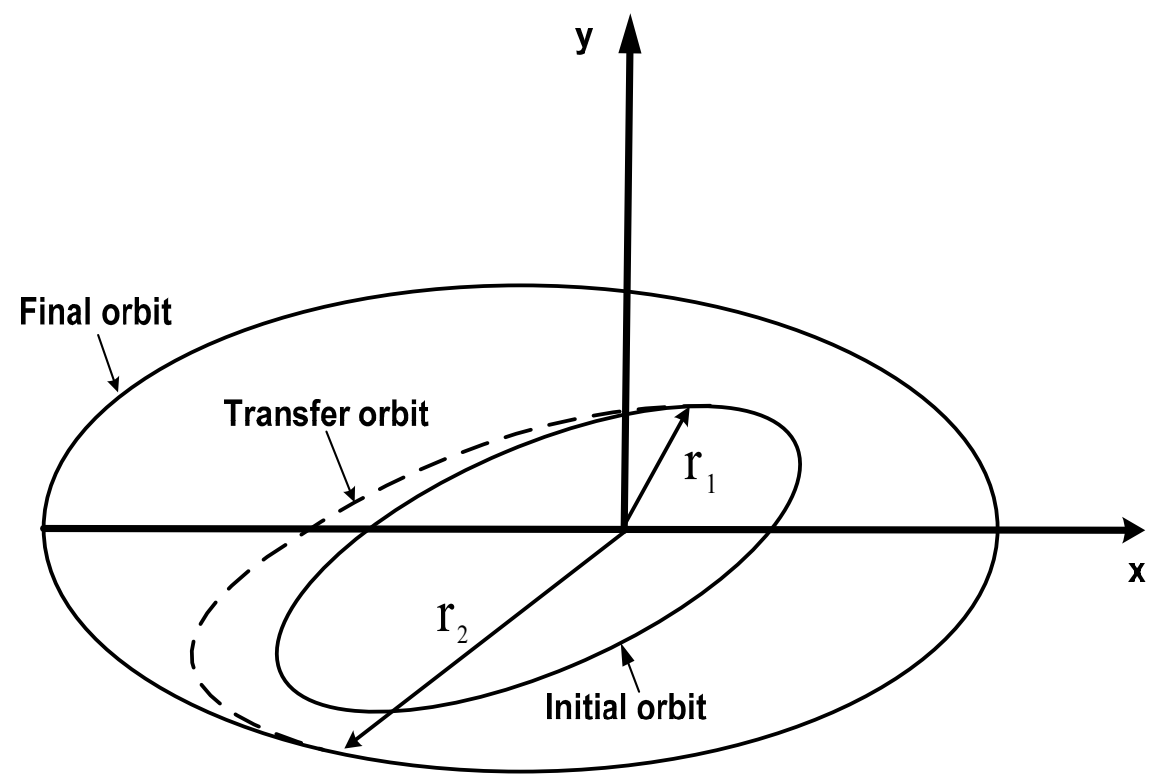

Fig. 1. Cotangential transfer. 
The solution of the present problem means the determination of:

1. The semi-major axis $\mathrm{a}_{\mathrm{T}}$ and the eccentricity $\mathrm{e}_{\mathrm{T}}$ of the transfer orbit.

2. The total impulse for the complete two impulse transfer at $r_{1}$ and $r_{2}$ which gives minimum velocity increment. Some numerical applications are also included.

\section{Necessary Tangency Conditions}

Continuity in position requires that $r_{1}=r_{T_{1}}$, where $r_{T_{1}}$ is the magnitude of the radius vector at the terminal 1 on the transfer orbit. Therefore, in more explicit form:

$$
\frac{p_{1}}{1+e_{1} \cos \left(f+\varphi_{1}\right)}=\frac{p_{T}}{1+e_{T} \cos \left(f+\varphi_{T}\right)}
$$

where 0

$\mathrm{p} \equiv$ the orbital parameter, $\mathrm{e} \equiv$ the orbital eccentricity, $\varphi \equiv$ the angle between arbitrary reference line and periapsis. If $\varphi_{1}=0$, the phase angles are with respect to periapsis of the initial orbit and $f \equiv$ the true anomaly.

Let

$$
\widetilde{\mathrm{p}} \equiv \frac{1}{\mathrm{p}} \quad ; \quad \widetilde{\mathrm{q}} \equiv \frac{\mathrm{e}}{\mathrm{p}}=\mathrm{e} \widetilde{\mathrm{p}} .
$$

From Equation (1) we have:

$$
\widetilde{\mathrm{q}}_{\mathrm{T}} \cos \left(\mathrm{f}+\varphi_{\mathrm{T}}\right)-\widetilde{\mathrm{q}}_{1} \cos \left(\mathrm{f}+\varphi_{1}\right)=-\left(\widetilde{\mathrm{p}}_{\mathrm{T}}-\widetilde{\mathrm{p}}_{1}\right),
$$

squaring this equation and then adding the zero value:

$$
\left\{2 \widetilde{\mathrm{q}}_{1} \widetilde{\mathrm{q}}_{\mathrm{T}} \sin \left(\mathrm{f}+\varphi_{1}\right) \sin \left(\mathrm{f}+\varphi_{\mathrm{T}}\right)\right\}-\left\{2 \widetilde{\mathrm{q}}_{1} \widetilde{\mathrm{q}}_{\mathrm{T}} \sin \left(\mathrm{f}+\varphi_{1}\right) \sin \left(\mathrm{f}+\varphi_{\mathrm{T}}\right)\right\}
$$

to the left side of the resulting equation we get :

$$
\widetilde{\mathrm{q}}_{\mathrm{T}}^{2}+\widetilde{\mathrm{q}}_{1}^{2}-2 \widetilde{\mathrm{q}}_{1} \widetilde{\mathrm{q}}_{\mathrm{T}} \cos \left(\varphi_{\mathrm{T}}-\varphi_{1}\right)-\left(\widetilde{\mathrm{q}}_{\mathrm{T}} \sin \left(\mathrm{f}+\varphi_{\mathrm{T}}\right)-\widetilde{\mathrm{q}}_{1} \sin \left(\mathrm{f}+\varphi_{1}\right)\right)^{2}=\left(\widetilde{\mathrm{p}}_{\mathrm{T}}-\widetilde{\mathrm{p}}_{1}\right)^{2}
$$

Now, the continuity in velocities requires that: 


$$
\frac{1}{\mathrm{r}_{1}^{2}} \frac{\mathrm{dr}_{1}}{\mathrm{df}}=\frac{1}{\mathrm{r}_{\mathrm{T}_{1}}^{2}} \frac{\mathrm{dr}_{\mathrm{T}_{1}}}{\mathrm{df}}
$$

By Equations (1) and (5) we obtain:

$$
\widetilde{\mathrm{q}}_{\mathrm{T}} \sin \left(\mathrm{f}+\varphi_{\mathrm{T}}\right)-\widetilde{\mathrm{q}}_{1} \sin \left(\mathrm{f}+\varphi_{1}\right)=0,
$$

therefore Equation (4) becomes:

$$
\widetilde{\mathrm{q}}_{\mathrm{T}}^{2}+\widetilde{\mathrm{q}}_{1}^{2}-2 \widetilde{\mathrm{q}}_{1} \widetilde{\mathrm{q}}_{\mathrm{T}} \cos \left(\varphi_{\mathrm{T}}-\varphi_{1}\right)=\left(\widetilde{\mathrm{p}}_{\mathrm{T}}-\widetilde{\mathrm{p}}_{1}\right)^{2} .
$$

In similar way, we have at the second or arrival terminal:

$$
\widetilde{\mathrm{q}}_{\mathrm{T}}^{2}+\widetilde{\mathrm{q}}_{2}^{2}-2 \widetilde{\mathrm{q}}_{2} \widetilde{\mathrm{q}}_{\mathrm{T}} \cos \left(\varphi_{\mathrm{T}}-\varphi_{2}\right)=\left(\widetilde{\mathrm{p}}_{\mathrm{T}}-\widetilde{\mathrm{p}}_{2}\right)^{2} .
$$

Equations (7) and (8) are system of two equations in the three unknowns $\widetilde{\mathrm{p}}_{\mathrm{T}}, \widetilde{\mathrm{q}}_{\mathrm{T}}$ and $\varphi_{\mathrm{T}}$.

The parameters $\widetilde{\mathrm{p}}_{\mathrm{i}}, \widetilde{\mathrm{q}}_{\mathrm{i}}$ and $\varphi_{\mathrm{i}} ; \mathrm{i}=1,2$ are known from the characteristics of the initial and final orbits. These equations can be written in the alternative form as follows. Since

$$
\cos \left(\varphi_{\mathrm{T}}-\varphi_{2}\right)=\cos \left(\varphi_{\mathrm{T}}-\varphi_{1}\right) \cos \left(\varphi_{2}-\varphi_{1}\right)+\sin \left(\varphi_{\mathrm{T}}-\varphi_{1}\right) \sin \left(\varphi_{2}-\varphi_{1}\right) .
$$

From Equation (7) we have:

$$
\cos \left(\varphi_{\mathrm{T}}-\varphi_{1}\right)=\frac{\left(\widetilde{\mathrm{q}}_{\mathrm{T}}^{2}+\widetilde{\mathrm{q}}_{1}^{2}\right)-\left(\widetilde{\mathrm{p}}_{\mathrm{T}}-\widetilde{\mathrm{p}}_{1}\right)^{2}}{2 \widetilde{\mathrm{q}}_{\mathrm{T}} \widetilde{\mathrm{q}}_{1}}
$$

and

$$
\sin \left(\varphi_{\mathrm{T}}-\varphi_{1}\right)= \pm \frac{\left(4 \widetilde{\mathrm{q}}_{\mathrm{T}}^{2} \widetilde{\mathrm{q}}_{1}^{2}-\left[\left(\widetilde{\mathrm{q}}_{\mathrm{T}}^{2}+\widetilde{\mathrm{q}}_{1}^{2}\right)-\left(\widetilde{\mathrm{p}}_{\mathrm{T}}-\widetilde{\mathrm{p}}_{1}\right)^{2}\right]^{2}\right)^{1 / 2}}{2 \widetilde{\mathrm{q}}_{\mathrm{T}} \widetilde{\mathrm{q}}_{1}} .
$$

Using these two equations together with Equation (8) [for $\left.\cos \left(\varphi_{\mathrm{T}}-\varphi_{2}\right)\right]$ into Equation (9), and then squaring we get:

$$
\begin{aligned}
F \equiv & \left\{\frac{\left(\widetilde{\mathrm{q}}_{\mathrm{T}}^{2}+\widetilde{\mathrm{q}}_{2}^{2}\right)-\left(\widetilde{\mathrm{p}}_{\mathrm{T}}-\widetilde{\mathrm{p}}_{2}\right)^{2}}{\widetilde{\mathrm{q}}_{2} \sin \left(\varphi_{2}-\varphi_{1}\right)}-\frac{\left(\widetilde{\mathrm{q}}_{\mathrm{T}}^{2}+\widetilde{\mathrm{q}}_{1}^{2}\right)-\left(\widetilde{\mathrm{p}}_{\mathrm{T}}-\widetilde{\mathrm{p}}_{1}\right)^{2}}{\widetilde{\mathrm{q}}_{1} \tan \left(\varphi_{2}-\varphi_{1}\right)}\right\}^{2} \\
& -\frac{4 \widetilde{\mathrm{q}}_{\mathrm{T}}^{2} \widetilde{\mathrm{q}}_{1}^{2}-\left\{\left(\widetilde{\mathrm{q}}_{\mathrm{T}}^{2}+\widetilde{\mathrm{q}}_{1}^{2}\right)-\left(\widetilde{\mathrm{p}}_{\mathrm{T}}-\widetilde{\mathrm{p}}_{1}\right)^{2}\right\}^{2}}{\widetilde{\mathrm{q}}_{1}^{2}}=0
\end{aligned}
$$


Note that $\mathrm{F}=\mathrm{F}\left(\widetilde{\mathrm{p}}_{\mathrm{T}}, \widetilde{\mathrm{q}}_{\mathrm{T}}\right)$, that is, the dimensional parameters of the transfer conic, are related by means of the this tangency function.

\section{Minimum Cotangential Velocity Increment Transfer}

From the energy equation, $v^{2}=\mu(2 / r-1 / a)$ and Equation (2) we have:

$$
v^{2}=\mu\left(\frac{2}{r}-\frac{\widetilde{p}^{2}-\widetilde{q}^{2}}{\widetilde{p}}\right) .
$$

In order to utilize Equation (11) it is necessary to evaluate the radius vector in terms of the auxiliary variables. To do this, consider the square of Equation (3), and then using Equation (3) $\widetilde{\mathrm{q}}_{\mathrm{T}} \cos \left(\mathrm{f}+\varphi_{\mathrm{T}}\right)$ and Equation (5) for $\widetilde{q}_{\mathrm{T}} \sin \left(f+\varphi_{\mathrm{T}}\right)$ we get:

$$
\frac{1}{\mathrm{r}_{1}}=\frac{\left(\widetilde{\mathrm{p}}_{\mathrm{T}}+\widetilde{\mathrm{p}}_{1}\right)}{2}-\frac{\widetilde{\mathrm{q}}_{\mathrm{T}}^{2}-\widetilde{\mathrm{q}}_{1}^{2}}{2\left(\widetilde{\mathrm{p}}_{\mathrm{T}}-\widetilde{\mathrm{p}}_{1}\right)} .
$$

Using Equations (11) and (12) we get:

$$
\mathrm{v}_{1}^{2}=\mu\left\{\widetilde{\mathrm{p}}_{\mathrm{T}}+\frac{\widetilde{\mathrm{p}}_{1} \widetilde{\mathrm{q}}_{\mathrm{T}}^{2}-\widetilde{\mathrm{q}}_{1}^{2} \widetilde{\mathrm{p}}_{\mathrm{T}}}{\widetilde{\mathrm{p}}_{1}\left(\widetilde{\mathrm{p}}_{1}-\widetilde{\mathrm{p}}_{\mathrm{T}}\right)}\right\} .
$$

Similarly, for the second terminal we have:

$$
\mathrm{v}_{2}^{2}=\mu\left\{\widetilde{\mathrm{p}}_{\mathrm{T}}+\frac{\widetilde{\mathrm{p}}_{2} \widetilde{\mathrm{q}}_{\mathrm{T}}^{2}-\widetilde{\mathrm{q}}_{2}^{2} \widetilde{\mathrm{p}}_{\mathrm{T}}}{\widetilde{\mathrm{p}}_{2}\left(\widetilde{\mathrm{p}}_{2}-\widetilde{\mathrm{p}}_{\mathrm{T}}\right)}\right\} .
$$

Since $r_{1}=r_{T_{1}}$, then we get from Equation (13) that:

$$
\mathrm{v}_{\mathrm{T}_{1}}^{2}=\mu\left\{\widetilde{\mathrm{p}}_{1}+\frac{\widetilde{\mathrm{p}}_{1} \widetilde{\mathrm{q}}_{\mathrm{T}}^{2}-\widetilde{\mathrm{q}}_{1}^{2} \widetilde{\mathrm{p}}_{\mathrm{T}}}{\widetilde{\mathrm{p}}_{\mathrm{T}}\left(\widetilde{\mathrm{p}}_{1}-\widetilde{\mathrm{p}}_{\mathrm{T}}\right)}\right\} .
$$

Similarly, for the second terminal we have:

$$
\mathrm{v}_{\mathrm{T}_{2}}^{2}=\mu\left\{\widetilde{\mathrm{p}}_{2}+\frac{\widetilde{\mathrm{p}}_{2} \widetilde{\mathrm{q}}_{\mathrm{T}}^{2}-\widetilde{\mathrm{q}}_{2}^{2} \widetilde{\mathrm{p}}_{\mathrm{T}}}{\widetilde{\mathrm{p}}_{\mathrm{T}}\left(\widetilde{\mathrm{p}}_{2}-\widetilde{\mathrm{p}}_{\mathrm{T}}\right)}\right\} .
$$


Furthermore, because the cotangential condition permits an algebraic addition of velocities, the total impulse for the complete two-impulse transfer becomes:

$$
\Delta \mathrm{V}=\left|\mathrm{v}_{\mathrm{T}_{2}}-\mathrm{v}_{2}\right|+\left|\mathrm{v}_{\mathrm{T}_{1}}-\mathrm{v}_{2}\right| .
$$

Using Equations (13), (14), (15) and (16) into the above equation we get:

$$
\Delta \mathrm{V}=\mu^{1 / 2}\left\{\left(\widetilde{\mathrm{p}}_{1}-\widetilde{\mathrm{p}}_{\mathrm{T}}+\frac{\widetilde{\mathrm{q}}_{\mathrm{T}}^{2}}{\widetilde{\mathrm{p}}_{\mathrm{T}}}-\frac{\widetilde{\mathrm{p}}_{1}^{2}}{\widetilde{\mathrm{p}}_{1}}\right)\left(\frac{\widetilde{\mathrm{p}}_{1}^{1 / 2}-\widetilde{\mathrm{p}}_{\mathrm{T}}^{1 / 2}}{\widetilde{\mathrm{p}}_{1}^{1 / 2}+\widetilde{\mathrm{p}}_{\mathrm{T}}^{1 / 2}}\right)\right\}^{1 / 2}+\mu^{1 / 2}\left\{\left(\widetilde{\mathrm{p}}_{2}-\widetilde{\mathrm{p}}_{\mathrm{T}}+\frac{\widetilde{\mathrm{q}}_{\mathrm{T}}^{2}}{\widetilde{\mathrm{p}}_{\mathrm{T}}}-\frac{\widetilde{\mathrm{q}}_{2}^{2}}{\widetilde{\mathrm{p}}_{2}}\right)\left(\frac{\widetilde{\mathrm{p}}_{2}^{1 / 2}-\widetilde{\mathrm{p}}_{\mathrm{T}}^{1 / 2}}{\widetilde{\mathrm{p}}_{2}^{1 / 2}+\widetilde{\mathrm{p}}_{\mathrm{T}}^{1 / 2}}\right)\right\}^{1 / 2}
$$

It should be noted that $\Delta \mathrm{V}=\Delta \mathrm{V}\left(\widetilde{\mathrm{p}}_{\mathrm{T}}, \widetilde{\mathrm{q}}_{\mathrm{T}}\right)$, since the parameters $\widetilde{\mathrm{p}}_{\mathrm{i}}, \widetilde{\mathrm{q}}_{i} ; \mathrm{i}=1,2$ are known from the characteristics of the initial and final orbits.

To obtain a cotangential transfer orbit with a minimum amount of impulse, Lagrange's rule must be introduced because of the intractability of the equations involved. The problem is therefore the minimization of $\Delta \mathrm{V}$ of Equation (17), subject to constraint $\mathrm{F}$, of Equation (10). To accomplish this result construct the objective function $\mathrm{M}$ defined by:

$$
\mathrm{M}=\Delta \mathrm{V}+\lambda \mathrm{F}
$$

and equate the partial derivatives with respect to $\widetilde{\mathrm{p}}_{\mathrm{T}}$ and $\widetilde{\mathrm{q}}_{\mathrm{T}}$ to zero, that is:

$$
\frac{\partial \mathrm{M}}{\partial \widetilde{\mathrm{p}}_{\mathrm{T}}}=\frac{\partial \Delta \mathrm{V}}{\partial \widetilde{\mathrm{p}}_{\mathrm{T}}}+\lambda \frac{\partial \mathrm{F}}{\partial \widetilde{\mathrm{p}}_{\mathrm{T}}}=0
$$

and

$$
\frac{\partial \mathrm{M}}{\partial \widetilde{\mathrm{q}}_{\mathrm{T}}}=\frac{\partial \Delta \mathrm{V}}{\partial \widetilde{\mathrm{q}}_{\mathrm{T}}}+\lambda \frac{\partial \mathrm{F}}{\partial \widetilde{\mathrm{q}}_{\mathrm{T}}}=0 .
$$

Eliminating $\lambda$ between these equations we get:

$$
\mathrm{G}\left(\widetilde{\mathrm{p}}_{\mathrm{T}}, \widetilde{\mathrm{q}}_{\mathrm{T}}\right) \equiv \frac{\partial \Delta \mathrm{V}}{\partial \widetilde{\mathrm{q}}_{\mathrm{T}}} \frac{\partial \mathrm{F}}{\partial \widetilde{\mathrm{p}}_{\mathrm{T}}}-\frac{\partial \Delta \mathrm{V}}{\partial \widetilde{\mathrm{p}}_{\mathrm{T}}} \frac{\partial \mathrm{F}}{\partial \widetilde{\mathrm{q}}_{\mathrm{T}}}=0
$$

which, with

$$
\mathrm{F}\left(\widetilde{\mathrm{p}}_{\mathrm{T}}, \widetilde{\mathrm{q}}_{\mathrm{T}}\right)=0
$$


provides a system of two algebraic equations in two unknowns whose zeros define the minimum velocity increment cotangential transfer conic.

\section{Numerical Developments}

\subsection{Partial Derivatives}

The partial derivatives involved in Equation (19), are:

$$
\begin{aligned}
& \frac{\partial \mathrm{F}}{\partial \widetilde{\mathrm{p}}_{\mathrm{T}}}=4 \mathrm{~F}_{1}\left\{-\frac{\left(\widetilde{\mathrm{p}}_{\mathrm{T}}-\widetilde{\mathrm{p}}_{2}\right)}{\widetilde{\mathrm{q}}_{2} \sin \left(\varphi_{2}-\varphi_{1}\right)}+\frac{\left(\widetilde{\mathrm{p}}_{\mathrm{T}}-\widetilde{\mathrm{p}}_{1}\right)}{\widetilde{\mathrm{q}}_{1} \tan \left(\varphi_{2}-\varphi_{1}\right)}\right\}+\frac{4}{\widetilde{\mathrm{q}}_{1}^{2}}\left(\widetilde{\mathrm{p}}_{\mathrm{T}}-\widetilde{\mathrm{p}}_{1}\right)\left\{\left(\widetilde{\mathrm{q}}_{\mathrm{T}}^{2}+\widetilde{\mathrm{q}}_{1}^{2}\right)+\left(\widetilde{\mathrm{p}}_{\mathrm{T}}-\widetilde{\mathrm{p}}_{1}\right)^{2}\right\} \\
& \frac{\partial \mathrm{F}}{\partial \widetilde{\mathrm{q}}_{\mathrm{T}}}=4 \mathrm{~F}_{1} \widetilde{\mathrm{q}}_{\mathrm{T}}\left\{\frac{1}{\widetilde{\mathrm{q}}_{2} \sin \left(\varphi_{2}-\varphi_{1}\right)}-\frac{1}{\widetilde{\mathrm{q}}_{1} \tan \left(\varphi_{2}-\varphi_{1}\right)}\right\}+\frac{4 \widetilde{\mathrm{q}}_{\mathrm{T}}}{\widetilde{\mathrm{q}}_{1}^{2}}\left\{\widetilde{\mathrm{q}}_{\mathrm{T}}^{2}-\widetilde{\mathrm{q}}_{1}^{2}+\left(\widetilde{\mathrm{p}}_{\mathrm{T}}-\widetilde{\mathrm{p}}_{1}\right)^{2}\right\} \\
& \mathrm{F}_{1}=\left\{\frac{\left(\tilde{\mathrm{q}}_{\mathrm{T}}^{2}+\widetilde{\mathrm{q}}_{2}^{2}\right)-\left(\widetilde{\mathrm{p}}_{\mathrm{T}}-\widetilde{\mathrm{p}}_{2}\right)^{2}}{\widetilde{\mathrm{q}}_{2} \sin \left(\varphi_{2}-\varphi_{1}\right)}-\frac{\left(\widetilde{\mathrm{q}}_{\mathrm{T}}^{2}+\widetilde{\mathrm{q}}_{1}^{2}\right)-\left(\widetilde{\mathrm{p}}_{\mathrm{T}}-\widetilde{\mathrm{p}}_{1}\right)^{2}}{\widetilde{\mathrm{q}}_{1} \tan \left(\varphi_{2}-\varphi_{1}\right)}\right\} \\
& \frac{\partial \Delta \mathrm{V}}{\partial \widetilde{\mathrm{p}}_{\mathrm{T}}}=\sqrt{\mu} \sum_{\mathrm{i}=1}^{2} \mathrm{Q}_{\mathrm{i}} \quad ; \quad \frac{\partial \Delta \mathrm{V}}{\partial \widetilde{\mathrm{q}}_{\mathrm{T}}}=\sqrt{\mu} \sum_{\mathrm{i}=1}^{2} \mathrm{~T}_{\mathrm{i}} \\
& \mathrm{Q}_{\mathrm{i}}=\frac{1}{2 \mathrm{G}}\left\{1-\frac{1}{4}\left(\frac{\widetilde{\mathrm{p}}_{\mathrm{i}}}{\widetilde{\mathrm{p}}_{\mathrm{T}}}\right)^{1 / 2}-\left(\frac{\widetilde{\mathrm{q}}_{\mathrm{T}}}{\widetilde{\mathrm{p}}_{\mathrm{T}}}\right)^{2}\left(\frac{\widetilde{\mathrm{p}}_{\mathrm{i}}+\widetilde{\mathrm{p}}_{\mathrm{T}}-\left(\widetilde{\mathrm{p}}_{\mathrm{i}} \widetilde{\mathrm{p}}_{\mathrm{T}}\right)^{1 / 2} / 2}{\widetilde{\mathrm{p}}_{\mathrm{i}}-\widetilde{\mathrm{p}}_{\mathrm{T}}}\right)+\frac{\left(\widetilde{\mathrm{q}}_{\mathrm{T}}^{2} / \widetilde{\mathrm{p}}_{\mathrm{T}}-\widetilde{\mathrm{q}}_{\mathrm{i}}^{2} / \mathrm{p}_{\mathrm{i}}\right)}{\left(\widetilde{\mathrm{p}}_{\mathrm{i}}-\widetilde{\mathrm{p}}_{\mathrm{T}}\right)^{2}}\left(1-\frac{1}{4}\left(\frac{\widetilde{\mathrm{p}}_{\mathrm{i}}}{\widetilde{\mathrm{p}}_{\mathrm{T}}}\right)^{1 / 2} \times\right.\right. \\
& \left.\left.\left.\times\left(\widetilde{\mathrm{p}}_{\mathrm{i}}-\widetilde{\mathrm{p}}_{\mathrm{T}}\right)+\widetilde{\mathrm{p}}_{\mathrm{i}}+\widetilde{\mathrm{p}}_{\mathrm{T}}-\frac{\left(\widetilde{\mathrm{p}}_{\mathrm{i}} \widetilde{\mathrm{p}}_{\mathrm{T}}\right)}{2}\right)\right)\right\} \quad ; \quad \mathrm{i}=1,2 . \\
& \mathrm{T}_{\mathrm{i}}=\frac{\widetilde{\mathrm{q}}_{\mathrm{T}}}{\mathrm{G}_{1} \widetilde{\mathrm{p}}_{\mathrm{T}}}\left\{\frac{\widetilde{\mathrm{p}}_{\mathrm{i}}+\widetilde{\mathrm{p}}_{\mathrm{T}}-\left(\widetilde{\mathrm{p}}_{\mathrm{i}} \widetilde{\mathrm{p}}_{\mathrm{T}}\right)^{1 / 2}}{\widetilde{\mathrm{p}}_{\mathrm{i}}-\widetilde{\mathrm{p}}_{\mathrm{T}}}\right\} ; \mathrm{i}=1,2 \\
& \mathrm{G}_{\mathrm{i}}=\left\{\widetilde{\mathrm{p}}_{\mathrm{i}}+\widetilde{\mathrm{p}}_{\mathrm{T}}-\left(\widetilde{\mathrm{p}}_{\mathrm{i}} \widetilde{\mathrm{p}}_{\mathrm{T}}\right)^{1 / 2} / 2+\left(\widetilde{\mathrm{p}}_{\mathrm{i}}+\widetilde{\mathrm{p}}_{\mathrm{T}}-\left(\widetilde{\mathrm{p}}_{\mathrm{i}} \widetilde{\mathrm{p}}_{\mathrm{T}}\right)^{1 / 2} / 2\right)\left(\widetilde{\mathrm{q}}_{\mathrm{T}}^{2} / \widetilde{\mathrm{p}}_{\mathrm{T}}-\widetilde{\mathrm{q}}_{\mathrm{i}}^{2} / \widetilde{\mathrm{p}}_{\mathrm{T}}\right) /\left(\widetilde{\mathrm{p}}_{\mathrm{i}}-\widetilde{\mathrm{p}}_{\mathrm{T}}\right)\right\}^{1 / 2} ; \mathrm{i}=1,2
\end{aligned}
$$


Now, Equations (19) and (20) are ready to be solved by Newton's iterative method for two simultaneous nonlinear equations illustrated in the following section.

\subsection{Newton's Iterative Solution for Two Simultaneous Nonlinear Equations}

Consider the two simultaneous nonlinear equation:

$$
\mathrm{U}(\mathrm{x}, \mathrm{y})=0 ; \mathrm{V}(\mathrm{x}, \mathrm{y})=0
$$

in two unknowns $\mathrm{x}$ and $\mathrm{y}$.

Let $\left(\mathrm{x}_{0}, \mathrm{y}_{0}\right)$ be an approximation to a root $(\xi, \eta)$ of the system of Equations (21). Generate successive approximations from the recursion formulae:

$$
\begin{aligned}
& \mathrm{x}_{\mathrm{i}+1}=\mathrm{x}_{i}-\left\{\left(\mathrm{U} \frac{\partial \mathrm{V}}{\partial \mathrm{y}}-\mathrm{V} \frac{\partial \mathrm{U}}{\partial \mathrm{y}}\right) / \mathrm{J}\right\}_{\mathrm{i}}=\mathrm{x}_{\mathrm{i}}-\delta \mathrm{x} ; \mathrm{i}=0,1, \cdots \\
& \mathrm{y}_{\mathrm{i}+1}=\mathrm{y}_{\mathrm{i}}-\left\{\left(\mathrm{V} \frac{\partial \mathrm{U}}{\partial \mathrm{x}}-\mathrm{U} \frac{\partial \mathrm{U}}{\partial \mathrm{x}}\right) / \mathrm{J}\right\}_{\mathrm{i}}=\mathrm{y}_{\mathrm{i}}-\delta \mathrm{y} ; \mathrm{i}=0,1,2, \ldots
\end{aligned}
$$

the Jacobin of the system is:

$$
\mathrm{J}(\mathrm{U}, \mathrm{V})=\frac{\partial \mathrm{U}}{\partial \mathrm{x}} \frac{\partial \mathrm{V}}{\partial \mathrm{y}}-\frac{\partial \mathrm{V}}{\partial \mathrm{x}} \frac{\partial \mathrm{U}}{\partial \mathrm{y}},
$$

where all the functions involved are to be evaluated at $\left(\mathrm{x}_{\mathrm{i}}, \mathrm{y}_{\mathrm{i}}\right)$.

When the iteration converges, it converges quadratically. A set of conditions sufficient to ensure convergence is the following:

1. $\mathrm{U}$ and $\mathrm{V}$ and all their derivatives through second order are continuous and bounded in a region $\mathrm{R}$ containing $(\xi, \eta)$.

2. The Jacobin $\mathrm{J}(\mathrm{U}, \mathrm{V})$ does not vanish in $\mathrm{R}$.

3. The initial approximation $\left(\mathrm{x}_{0}, \mathrm{y}_{0}\right)$ is chosen sufficiently close to the $\operatorname{root}(\xi, \eta)$.

The iterative process may be stopped by the condition that:

$$
\operatorname{Max}(|\mathrm{U}|,|\mathrm{V}|,|\delta \mathrm{x}|,|\delta \mathrm{y}|) \leq \text { Tol. }
$$


where Tol. given tolerance.

\subsection{Numerical Examples}

For both examples we used for Tol the value $10^{-6}$.

1. Given: $\widetilde{\mathrm{p}}_{1}=1.0 \mathrm{ER} ; \widetilde{\mathrm{p}}_{2}=1.8 \mathrm{ER} ; \widetilde{\mathrm{q}}_{1}=0.4 \mathrm{ER} ; \widetilde{\mathrm{q}}_{2}=0.2 \mathrm{ER}$

$$
\varphi_{1}=0^{\circ} \quad ; \quad \varphi_{2}=70^{\circ} \quad ; \mu=1 .
$$

Initial values : $\widetilde{\mathrm{p}}_{\mathrm{T} 0}=0.3 \mathrm{ER} \quad ; \quad \widetilde{\mathrm{q}}_{\mathrm{T} 0}=1.0 \mathrm{ER}$

ER is the Earth's radius

Find: $\mathrm{a}_{\mathrm{T}}, \mathrm{e}_{\mathrm{T}}$ and $\Delta \mathrm{V}$

- With initial conditions $\widetilde{\mathrm{p}}_{\mathrm{T} 0}, \widetilde{\mathrm{q}}_{\mathrm{T} 0}$, and the adopted tolerance, the solutions are:

$$
\widetilde{\mathrm{p}}_{\mathrm{T}}=1.4348 \text { ER and } \widetilde{\mathrm{q}}_{\mathrm{T}}=0.259522 \text { ER, consequently }
$$

$\mathrm{e}_{\mathrm{T}}=0.180877$ that the transfer orbit is elliptic ; $\mathrm{a}_{\mathrm{T}}=1.48333 \mathrm{ER}=$ $9460.8809 \mathrm{~km}$

and $\Delta \mathrm{V}=0.370664 \frac{\mathrm{ER}}{\mathrm{TU}}=2.93023 \mathrm{~km} / \mathrm{sec}$

2. Given: $\widetilde{\mathrm{p}}_{1}=0.6 \mathrm{ER} ; \widetilde{\mathrm{p}}_{2}=0.6 \mathrm{ER} ; \widetilde{\mathrm{q}}_{1}=0.8 \mathrm{ER} ; \widetilde{\mathrm{q}}_{2}=0.8 \mathrm{ER}$

$$
\varphi_{1}=60^{\circ} \quad ; \quad \varphi_{2}=30^{\circ} \quad ; \quad \mu=1 .
$$

Initial values : $\widetilde{\mathrm{p}}_{\mathrm{T} 0}=1.0 \mathrm{ER} \quad ; \quad \widetilde{\mathrm{q}}_{\mathrm{T} 0}=1.0 \mathrm{ER}$

Find: $\mathrm{a}_{\mathrm{T}}, \mathrm{e}_{\mathrm{T}}$ and $\Delta \mathrm{V}$

- With initial conditions $\widetilde{\mathrm{p}}_{\mathrm{T} 0}, \widetilde{\mathrm{q}}_{\mathrm{T} 0}$, and the adopted tolerance, the solutions are:

$\widetilde{\mathrm{p}}_{\mathrm{T}}=4.41683$ ER and $\widetilde{\mathrm{q}}_{\mathrm{T}}=0$, consequently $\mathrm{e}_{\mathrm{T}}=0$, that the transfer orbit is circular $\mathrm{a}_{\mathrm{T}}=4.41683 \mathrm{ER}=28171.14375 \mathrm{~km}$ and $\Delta \mathrm{V}=3.0021 \frac{\mathrm{ER}}{\mathrm{TU}}=23.73270 \mathrm{~km} / \mathrm{sec}$ 


\section{Conclusion}

In this paper, an efficient two impulses cotangential orbital transfer maneuvers is developed its efficiency is due some factors:

1. It is universal in the sense that, both the inner and transfer orbits are of arbitrary shapes (circular, elliptic, parabolic or hyperbolic). This a an important factor due to the fact that, during space mission all types of the two body motion appear. In addition, in some systems, the type of an orbit is occasionally changed by perturbing forces during finite interval of time. Consequently universal formulations are desperately needed so that orbit transfers will be free of the troubles, since a single functional representation suffices to describe all possible states.

2. As far as the impulses are concerned, the developed transfer maneuvers is general in the sense that, the two impulses are applied tangentially at two general points $r_{1}$ and $r_{2}$ not necessarily to be applied at the apsides.

3. It is a typical constraint minimization problem, by its solution we can The solution of the determination of, the semi-major axis $\mathrm{a}_{\mathrm{T}}$ and the eccentricity $\mathrm{e}_{\mathrm{T}}$ of the transfer orbit, and the total impulse for the complete two impulse transfer at $r_{1}$ and $r_{2}$ which gives minimum velocity increment. Some numerical applications are also included.

\section{Acknowledgments}

This work has been carried out as part of King Abdulaziz University sponsored research project $156 / 427$.

\section{References}

[1] Chobotov, V.A. (1996) Orbital Mechanics, $2^{\text {nd }}$ ed., AIAA Education Series.

[2] Lin, L.Z.W. (2007) Chinese Astronomy and Astrophysics, 31: 288-295.

[3] Villac, B.F. and Scheeres, D.J. (2003) Journal of Guidance, Control, Dynamics, 26(5) September-October: 750-757.

[4] Escobal, P.R. (1968) Methods of Astrodynamics, John Wiley \& Sons, Inc., New YorkLondon. 
مناور ات الانتقال المثالي التماسي

\section{محمد عادل شرف}

قسم العلوم الفلكية - كلية العلوم - جامعة الملك عبد العزيز

$$
\text { جدة - المدلكة العربية السعودية الية الهوله }
$$

المستخلص. تم في هذا البحث تشيد مناورات الانتقال المثالي

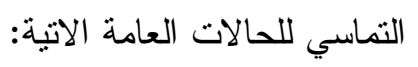

1- نوع كل من مدار الانتقال و المدار الداخلي افتر اضيًا.

r- كل من الدفعين يؤثز مماسياً عند أي نقطتين، وليس من

$$
\text { الضروري عند نقطي الأوج و الحضيض. }
$$

وعلية فإن حل المسألة هو تعين طول نصف المحور الأكبر

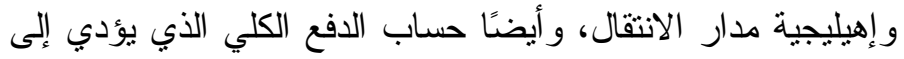
أقل تغير في السرعة. احتوي البحث على بعض التطبيقات العددية. 